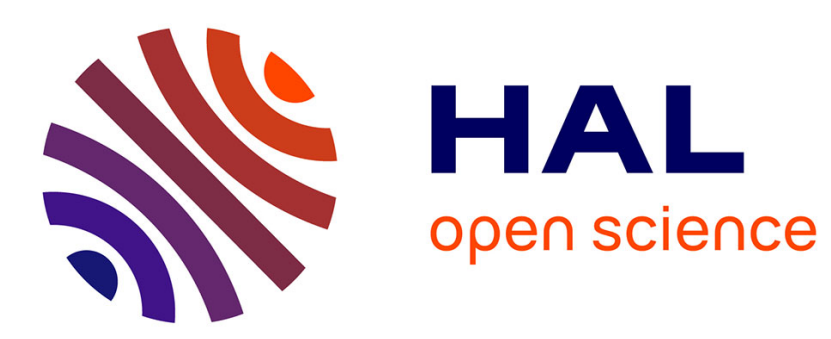

\title{
Fault detection based on interval analysis
}

Olivier Adrot, Hicham Janati-Idrissi, Didier Maquin

\section{To cite this version:}

Olivier Adrot, Hicham Janati-Idrissi, Didier Maquin. Fault detection based on interval analysis. 15th IFAC World Congress on Automatic Control, Jul 2002, Barcelonne, Spain. pp.CDROM. hal-00278211

\section{HAL Id: hal-00278211 \\ https://hal.science/hal-00278211}

Submitted on 9 May 2008

HAL is a multi-disciplinary open access archive for the deposit and dissemination of scientific research documents, whether they are published or not. The documents may come from teaching and research institutions in France or abroad, or from public or private research centers.
L'archive ouverte pluridisciplinaire HAL, est destinée au dépôt et à la diffusion de documents scientifiques de niveau recherche, publiés ou non, émanant des établissements d'enseignement et de recherche français ou étrangers, des laboratoires publics ou privés. 


\title{
FAULT DETECTION BASED ON INTERVAL ANALYSIS
}

\author{
O. Adrot, H. Janati-Idrissi, D. Maquin
}

\author{
Centre de Recherche en Automatique de Nancy - CNRS UMR 7039 \\ Institut National Polytechnique de Lorraine \\ 2, avenue de la Forêt de Haye - 54516 Vandouvre-lès-Nancy Cedex, France \\ Phone: (33) 383595959 - Fax: (33) 383595644 \\ \{Olivier.Adrot, hjanati,Didier.Maquin\}@ensem.inpl-nancy.fr
}

\begin{abstract}
This paper deals with a fault detection method taking into account model uncertainties described by bounded variables. A parity space approach is proposed, where the parity matrix depends on uncertain parameters. Since residuals represent a set of feasible behaviors, they therefore define a normal operation domain. In order to simplify its evaluation, residuals are linearized in bounded variables. This procedure generates an approximation, which can be enhanced by estimating bounds of uncertain parameters. Temporal dependencies between residuals are then taken into account in order to increase the precision of consistency tests. Copyright (C) 2002 IFAC
\end{abstract}

Keywords: fault detection, parity space, uncertain dynamic systems, intervals.

\section{INTRODUCTION}

Residual generation is a step of Fault Detection (F.D.) methods. It consists in structuring mathematical equations of a model in order to make this information exploitable in the form of indicators (called residuals) sensible to faults which must be detected. In this paper, the second section details a F.D. procedure in case of dynamic models, where uncertainties are assumed to be described by timevariant and bounded variables. A parity space method and associated consistency tests are developed. In order to simplify these tests by working on convex parallelotopes, a linearization procedure of residuals is proposed. The section 3 focuses on the problems caused by dependencies between bounded variables and reminds a method allowing to determine the characteristics representative of a parallelotope. The section 4 describes a method which estimates bounds of uncertainties in order to reduce the approximation due to the linearization procedure. During this step, dependencies between bounded variables are taken into account and this additional information is used to improve consistency test results. At last, an example illustrates our method in section 5 .

\section{RESIDUAL GENERATION}

\subsection{Model presentation}

Uncertain structured models take into account the lack of knowledge on a physical system by indicating which parameters are uncertain. These uncertainties are known as multiplicative ones since they straight affect model parameters. A set-membership approach being chosen, these uncertainties are described by normalized bounded variables, which bounds are equal to -1 and 1 . In fact, the components of the timevariant uncertain vector $\theta_{k}$ are represented by independent random variables $\theta_{k}^{i}$ with bounded realizations. Moreover, at two different instants $k$ and $t$, it is assumed that a same uncertainty is represented by two independent variables $\theta_{k}^{i}$ and $\theta_{t}^{i}$ with the same bounds.

In the fault free case, only dynamic systems described by linear discrete state equations are considered. Notice that uncertainties may affect all the matrices $\boldsymbol{A}, \boldsymbol{B}$ and $\boldsymbol{C}$ of the following model:

$$
\begin{cases}\boldsymbol{x}_{k+1}=\boldsymbol{A}\left(\boldsymbol{\theta}_{k}\right) \boldsymbol{x}_{k}+\boldsymbol{B}\left(\boldsymbol{\theta}_{k}\right) \boldsymbol{u}_{k}, & \boldsymbol{x} \in \mathbb{R}^{s_{x}}, \theta \in \mathbb{R}^{s_{\theta}} \\ \boldsymbol{y}_{k}=\boldsymbol{C}\left(\boldsymbol{\theta}_{k}\right) \boldsymbol{x}_{k} & \boldsymbol{y} \in \mathbb{R}^{s_{y}}, \boldsymbol{u} \in \mathbb{R}^{s_{u}}\end{cases}
$$

The terms $\boldsymbol{x}_{k}, \boldsymbol{u}_{k}$ and $\boldsymbol{y}_{k}, k \in\{1, \mathrm{~K}, h\}$, respectively define the state, actuator input and sensor output vectors at time $k$. The vector $\theta_{k}$ contains all uncertain parameters affecting this model and the matrices $\boldsymbol{A}, \boldsymbol{B}$ and $\boldsymbol{C}$ are assumed to be linear in uncertainties.

\subsection{Parity space approach}

A major drawback of interval analysis is its explosive nature in case of set-membership recursive systems. In order to avoid this problem known as wrapping effect, a parity space approach is chosen. This approach consists in formulating the dynamic model equations in the form of algebraic relations. By stacking sensory observations on a chosen time horizon $s$, a static representation is obtained where it is no need to integrate model equations in order to generate residuals (Adrot et al., 1999, 2000a): 


$$
\begin{gathered}
\boldsymbol{O}_{s}\left(\boldsymbol{\theta}_{k, s}\right) \boldsymbol{x}_{k, s}=\boldsymbol{H}_{s}\left(\boldsymbol{\theta}_{k, s-1}\right)\left[\begin{array}{c}
\boldsymbol{u}_{k, s-1} \\
\boldsymbol{y}_{k, s}
\end{array}\right], \\
\underbrace{\boldsymbol{z}_{k, s}}_{\boldsymbol{z} \in\{\boldsymbol{u}, \boldsymbol{x}, \boldsymbol{y}, \boldsymbol{\theta}\}}=\left[\begin{array}{r}
\boldsymbol{z}_{k} \\
\mathrm{M} \\
\boldsymbol{z}_{k+s}
\end{array}\right], \begin{array}{l}
\boldsymbol{O}_{s} \in \mathbb{R}^{\left\{(s+1) s_{y}+s s_{x}\right\} \times(s+1) s_{x}} \\
\boldsymbol{H}_{s} \in \mathbb{R}^{\left\{(s+1) s_{y}+s s_{x}\right\} \times\left\{s s_{u}+(s+1) s_{y}\right\}}
\end{array}
\end{gathered}
$$

In the previous equality (2), the term on the left depends on unknown state variables whereas the term on the right groups together measured outputs and inputs. Moreover both distribution matrices $\boldsymbol{O}_{s}$ and $\boldsymbol{H}_{s}$ depend on uncertainties. Now, the objective is to eliminate the unknown vector $\boldsymbol{x}_{k, s}$ in order to build some residuals which use the redundancy of the previous model (1) and which can be evaluated. Thus, an uncertain parity matrix $\boldsymbol{W}\left(\boldsymbol{\theta}_{k, s}\right)$ orthogonal to $\boldsymbol{O}_{s}\left(\boldsymbol{\theta}_{k, s}\right)$ is searched: $\boldsymbol{W}\left(\boldsymbol{\theta}_{k, s}\right) \boldsymbol{O}_{s}\left(\boldsymbol{\theta}_{k, s}\right)=0$.

The existence condition of this parity matrix and its symbolic expression are given in (Adrot et al., 2000b); moreover, $\boldsymbol{W}\left(\boldsymbol{\theta}_{k, s}\right)$ can always be written in the form of a polynomial matrix in uncertainties. Now, after multiplying the static form (2) by $\boldsymbol{W}\left(\theta_{k, s}\right)$, the expression of the residual vector $\boldsymbol{r}_{k}$ is deduced:

$$
\boldsymbol{r}_{k}\left(\boldsymbol{\theta}_{k, s}\right)=\boldsymbol{P}\left(\boldsymbol{\theta}_{k, s}\right)\left[\begin{array}{c}
\boldsymbol{u}_{k, s-1} \\
\boldsymbol{y}_{k, s}
\end{array}\right], \begin{aligned}
& \boldsymbol{r}_{k} \in \mathbb{R}^{s_{r}} \\
& \boldsymbol{\theta}_{k, s} \in \mathbb{R}^{(s+1) s_{\theta}},
\end{aligned}
$$

where $\boldsymbol{P}\left(\theta_{k, s}\right)$ is a polynomial matrix too. Moreover, this expression depends on all the uncertainties which initially affect the state representation (1).

\subsection{Consistency test}

At a given instant $k$, the physical system normally operates if at least one particular value $\theta_{0} \in \mathbb{R}^{(s+1) s_{\theta}}$ of the uncertain vector $\theta_{k, s}$ exists such that:

- the model is consistent with measurements, that implies $\boldsymbol{r}_{k}\left(\theta_{0}\right)=0$,

- $\theta_{0}$ is a feasible value in the sense that $\left\|\theta_{0}\right\|_{\infty} \leq 1$.

The value set $S\left(\boldsymbol{r}_{k}\right)$ of the residual vector defines all the feasible values of $\boldsymbol{r}_{k}$, which are consistent with the chosen model according to sensory observations and constraints $\left\|\theta_{k, s}\right\|_{\infty} \leq 1$. Thus, $S\left(\boldsymbol{r}_{k}\right)$ represents the normal operation domain of the monitored physical system and a fault is detected if the origin $O$ of the residual space does not belong to $S\left(\boldsymbol{r}_{k}\right)$.

Since an interval polynomial function is inclusion monotonic (Moore, 1979), the evaluation of $\boldsymbol{S}\left(\boldsymbol{r}_{k}\right)$ leads to a domain which necessarily contains $S\left(\boldsymbol{r}_{k}\right)$. Thus, the proposed method does not generate false alarms other than those due to the no-completeness of the model. Thus, if the model is initially complete (Armengol et al., 1999), an inconsistency necessarily guarantees the presence of a fault. On the contrary, a consistency does not assure the absence of a fault which may be masked by some uncertainties.

\subsection{Linearization procedure}

Since $\boldsymbol{r}_{k}$ (3) is non-linear in bounded variables $\theta_{k, s}$, to evaluate its value set $\boldsymbol{S}\left(\boldsymbol{r}_{k}\right)$ is very difficult. In order to simplify consistency tests, a procedure detailed in (Adrot et al., 2000b) allows to obtain a residual vector linear in uncertainties. The principle is to replace each monomial of bounded variables occurring in $\boldsymbol{r}_{k}$ by a new independent variable with an adequate value set. For example, monomials $\theta_{k}^{i} \theta_{k+1}^{i}$ and $\theta_{k}^{i^{2}}$ are replaced by $\mu_{k}^{j}$ and $0.5+0.5 \mu_{k}^{l}$, where $\mu_{k}^{j}$ and $\mu_{k}^{l}$ defines the $j^{\text {th }}$ and $l^{\text {th }}$ components of a normalized vector $\mu_{k}$. Thus, the dependence between these monomials is lost since $\mu_{k}^{j}$ and $\mu_{k}^{l}$ are considered as independent. In this way, the linearization is guaranteed in the sense that the value set of linearized residuals $\boldsymbol{r}_{\text {lin, } k}$ always includes the exact domain $S\left(\boldsymbol{r}_{k}\right)$ (Adrot et al., 2000b). Thus, $\mathrm{S}\left(\boldsymbol{r}_{\text {lin, }, k}\right)$ becomes a convex parallelotope, which can be easily evaluated as explained in section 3.3.

At last, by noting $\mu_{k}$ the vector composed of all normalized bounded variables contained in the linearized residual vector, $\boldsymbol{r}_{l i n, k} \in \mathbb{R}^{s_{r}}$ is written as follows where the matrix $\boldsymbol{R}_{\mu}$ and the vector $\boldsymbol{r}_{0}$ are linear in measurements:

$$
\boldsymbol{r}_{\text {lin, }, k}\left(\mu_{k}\right)=\boldsymbol{R}_{\mu}\left(\boldsymbol{y}_{k, s}, \boldsymbol{u}_{k, s-1}\right) \mu_{k}+\boldsymbol{r}_{0}\left(\boldsymbol{y}_{k, s}, \boldsymbol{u}_{k, s-1}\right)
$$

\section{DEPENDENCE PROBLEM}

\subsection{Dependence between some components of $\boldsymbol{r}_{k}$}

The considered problem is that interval analysis does not take into account the dependence between several bounded variables (Moore, 1979). This comes from the fact that interval analysis works on their bounds where this dependence does not appear.

In case of a bounded vector field $\boldsymbol{f}(\boldsymbol{\theta})$ (like $\boldsymbol{r}_{\text {lin,k }}$ or $\boldsymbol{r}_{k}$ ), some bounded variables (called common variables) may occur in several of its components $f^{i}(\theta)$. If $\boldsymbol{f}(\theta)$ has no common variable, every function $f^{i}(\theta)$ is independent and the value set $S(f)$ leads to an axis-aligned orthotope in the space of components $f^{i}$. Nevertheless, if at least one common variable exists, dependencies between some $f^{i}$ make the shape of $S(f)$ more complicated (Adrot et al., 2000a). Thus, if all functions $f^{i}$ are linear in bounded variables (as for $\boldsymbol{r}_{l i n, k}$ ), then $S(f)$ is a convex parallelotope, i.e. a polytope delimited by two by two parallel hyperplanes. The following example, where the variables $\theta^{i}, i \in\{1, \ldots, 3\}$ are common, leads to the 
domain $S(f)$ represented in figure 1 :

$$
f^{1}=\theta^{1}+\theta^{2}+\theta^{3} \quad f^{2}=\theta^{1}-\theta^{2}+2 \theta^{3} .
$$

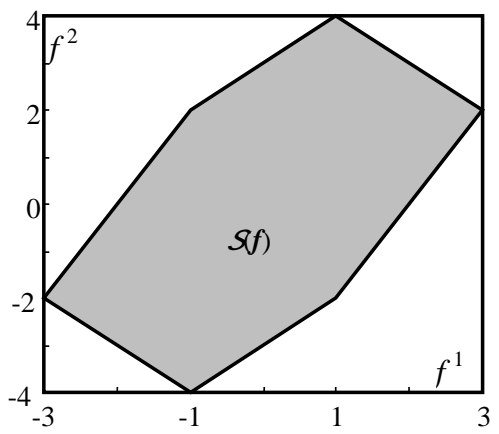

Fig 1. Value set of $\boldsymbol{f}$

\subsection{Temporal dependence}

The vector $\mu_{k}$ contains some bounded variables expressed at different instants on the time horizon $[k, k+s]$. In fact, residual vectors $\boldsymbol{r}_{l i n, k}\left(\mu_{k}\right)$ and $\boldsymbol{r}_{\text {lin, } k+t}\left(\mu_{k+t}\right), t \in\{1, \mathrm{~K}, s\}$, may depend on several identical bounded variables. For example, if $\mu_{k}=\left[\begin{array}{ll}\theta_{k}^{i} & \theta_{k+1}^{i}\end{array}\right]^{T}$, then both vectors $\mu_{k}$ and $\mu_{k+1}$ depend on the same variable $\theta_{k+1}^{i}$. These dependencies between delayed residuals due to identical uncertain parameters expressed at the same instant are interesting because they introduce new constraints on the normal operation field of the physical system and thus may increase the quality of the F.D. procedure. The objective is now to explain how using this information for parameter estimation and fault detection. Thus, by stacking the linearized residual vector $\boldsymbol{r}_{l i n, k}\left(\mu_{k}\right)$ (4) on the time horizon $[k, k+s]$, the following expression is obtained:

$$
\begin{gathered}
\boldsymbol{r}_{l i n, k, s}\left(\mu_{k, s}\right)=\boldsymbol{R}_{\mu, k, s}\left(\boldsymbol{y}_{k, k+2 s}, \boldsymbol{u}_{k, k+2 s-1}\right) \mu_{k, s}+ \\
\mathrm{K}+\boldsymbol{r}_{0, k, s}\left(\boldsymbol{y}_{k, k+2 s}, \boldsymbol{u}_{k, k+2 s-1}\right), \\
\text { with: } \boldsymbol{r}_{l i n, k, s}\left(\mu_{k, s}\right)=\left[\begin{array}{c}
\boldsymbol{r}_{l i n, k}\left(\mu_{k}\right) \\
\mathrm{M} \\
\boldsymbol{r}_{l i n, k+s}\left(\mu_{k+s}\right)
\end{array}\right], \boldsymbol{r}_{l i n, k, s} \in \mathbb{R}^{(s+1) s_{r}} \\
\mu_{k, s}=\left[\begin{array}{r}
\mu_{k} \\
\mathrm{M} \\
\mu_{k+s}
\end{array}\right], \\
\boldsymbol{R}_{\mu, k, s}(\boldsymbol{y}, \boldsymbol{u})=\left[\begin{array}{ccc}
\boldsymbol{R}_{\mu}\left(\boldsymbol{y}_{k, s}, \boldsymbol{u}_{k, s-1}\right) & 0 & 0 \\
0 & 0 & 0 \\
0 & 0 & \boldsymbol{R}_{\mu}\left(\boldsymbol{y}_{k+s, s}, \boldsymbol{u}_{k+s, s-1}\right)
\end{array}\right], \\
\boldsymbol{r}_{0, k, s}(\boldsymbol{y}, \boldsymbol{u})=\left[\begin{array}{c}
\boldsymbol{r}_{0}\left(\boldsymbol{y}_{k, s}, \boldsymbol{u}_{k, s-1}\right) \\
\mathrm{M} \\
\boldsymbol{r}_{0}\left(\boldsymbol{y}_{k+s, s}, \boldsymbol{u}_{k+s, s-1}\right)
\end{array}\right] .
\end{gathered}
$$

This horizon makes it possible to treat the temporal dependencies which can affect $\boldsymbol{r}_{\text {lin, }}$ whereas a higher value increases the delay in fault detection. For simplifying notations, symbols $\boldsymbol{u}$ and $\boldsymbol{y}$ referring to measurements will be omitted in the following. Moreover, the columns of $\boldsymbol{R}_{\mu, k, s}$ associated to the same bounded variables must be put together. For example, if $\mu_{k}^{i}=\theta_{k}^{l} \theta_{k+1}^{l}$ and $\mu_{k}^{j}=\theta_{k+1}^{l} \theta_{k+2}^{l}$, then the columns associates to $\mu_{k+1}^{i}$ and $\mu_{k}^{j}$ are summed since $\mu_{k+1}^{i}=\mu_{k}^{j}$. Thus, a reduced bounded vector $v_{k, s}$ is built from $\mu_{k, s}$ and the residual vector becomes:

$$
\boldsymbol{r}_{l i n, k, s}\left(v_{k, s}\right)=\boldsymbol{R}_{v, k, s} v_{k, s}+\boldsymbol{r}_{0, k, s}, v_{k, s} \in \mathbb{R}^{s_{\mathrm{v}}} .
$$

\subsection{Strip constraint decomposition}

It is assumed that $\boldsymbol{R}_{v, k, s}$ is full column rank. This hypothesis is in no way restrictive. Indeed, if $\boldsymbol{R}_{v, k, s}$ is not full column rank, it can always be broken down into $\boldsymbol{R}_{\mathrm{v}, k, s}=\boldsymbol{R}_{a}^{T} \boldsymbol{R}_{b}$ where both matrices $\boldsymbol{R}_{a}$ and $\boldsymbol{R}_{b}$ are full row rank. In this case, (5) is multiplied by the pseudo-inverse $\boldsymbol{R}_{a}^{+}$of $\boldsymbol{R}_{a}^{T}$ and becomes:

$$
\left\{\begin{array}{l}
\boldsymbol{R}_{a}^{+} \boldsymbol{r}_{l i n, k, s}\left(v_{k, s}\right)=\boldsymbol{R}_{b} v_{k, s}+\boldsymbol{R}_{a}^{+} \boldsymbol{r}_{0, k, s} \\
\left(\boldsymbol{I}-\boldsymbol{R}_{a}^{T} \boldsymbol{R}_{a}^{+}\right) \boldsymbol{r}_{l i n, k, s}\left(v_{k, s}\right)=\left(\boldsymbol{I}-\boldsymbol{R}_{a}^{T} \boldsymbol{R}_{a}^{+}\right) \boldsymbol{r}_{0, k, s}
\end{array} .\right.
$$

The second equality corresponds to a deterministic relation and is carried out in a straightforward way, while the first one has the same form as (5).

The objective of this section is to remember a method making it possible to construct exactly $\boldsymbol{S}\left(\boldsymbol{r}_{l i n, k, s}\right)$. More precisely, since $\boldsymbol{r}_{l i n, k, s}$ is linear in bounded variables $v_{k, s}$, its value set is a convex parallelotope centered in $\boldsymbol{r}_{0, k, s}$. In other words, it is a domain delimited by two by two parallel hyperplanes (strip constraints) in the residual space. In fact, $\boldsymbol{S}\left(\boldsymbol{r}_{l i n, k, S}\right)$ can be described by the intersection of several strip constraints $\boldsymbol{S}^{i}$ : $\boldsymbol{S}\left(\boldsymbol{r}_{l i n, k, S}\right)=\mid \boldsymbol{S}^{i}$. For example, figure 2 shows a parallelotope perfectly defined by the intersection of the strip constraints $S^{i}, i \in\{1,2,3\}$.

In a general manner, $S^{i}$ is defined by a two sides inequality deduced from (5) describing two half spaces which frontiers are parallel:

$$
\mathrm{S}^{i}=\left\{\boldsymbol{r}_{l i n, k, S} /-k^{i}+\boldsymbol{h}^{i^{T}} \boldsymbol{r}_{0, k, s} \leq \boldsymbol{h}^{i^{T}} \boldsymbol{r}_{\text {lin }, k, s} \leq k^{i}+\boldsymbol{h}^{i} \boldsymbol{r}_{0, k, S}\right\},(6)
$$

where the parameters $k^{i}$ and $\boldsymbol{h}^{i}$ must be computed.

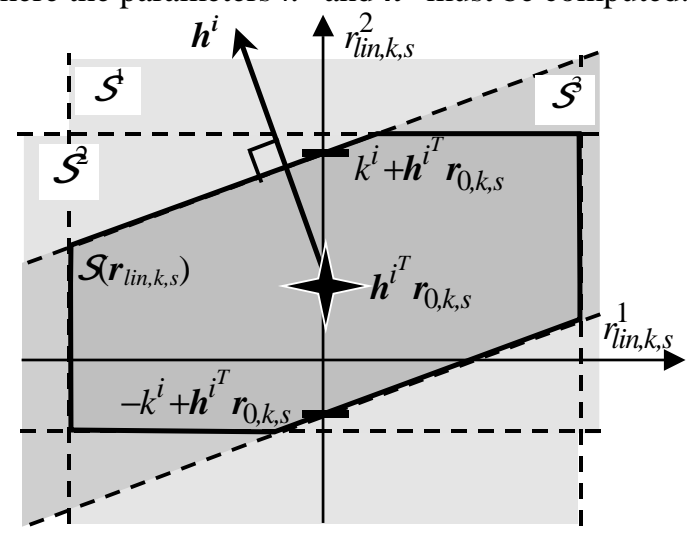

Fig 2. Strip constraint decomposition 
The scalar $k^{i}$ and the vector $\boldsymbol{h}^{i}$ respectively adjust the width and the direction of $S^{i}$, as shown in figure 2. The computation of $k^{i}$ and $\boldsymbol{h}^{i}$ uses the algorithm presented in (Ploix et al., 2000). Let us note by $\boldsymbol{e}^{l}$, $l \in\left\{1, \mathrm{~K}, s_{v}\right\}$, the vectors of the canonical basis of $\mathbb{R}^{s_{v}}$. Let us consider the $\mathbb{C}_{s_{v}}^{(s+1) s_{r}-1}$ matrices $\boldsymbol{R}_{v, k, s}^{i}$ built by combination of $(s+1) s_{r}-1$ different columns of the matrix $\boldsymbol{R}_{v, k, s}$ :

$$
\boldsymbol{R}_{\mathrm{v}, k, s}^{i}=\boldsymbol{R}_{\mathrm{v}, k, s}\left[\boldsymbol{e}^{i_{1}} \mathbf{K} \boldsymbol{e}^{i_{(s+1) s_{r}-1}}\right], \begin{aligned}
& i_{j} \in\left\{1, \mathrm{~K}, s_{v}\right\} \\
& j \in\left\{1, \mathrm{~K},(s+1) s_{r}-1\right\}
\end{aligned}{ }^{(7)}
$$

If this matrix is full column rank, then a new strip constraint $S^{i}$ exists and is determined by:

$$
\boldsymbol{h}^{i^{T}} \boldsymbol{R}_{\mathrm{v}, k, s}^{i}=0 \text { and } k^{i}=\left\|\boldsymbol{h}^{i^{T}} \boldsymbol{R}_{\mathrm{v}, k, s}\right\|_{1} .
$$

At the end, $\boldsymbol{S}\left(\boldsymbol{r}_{l i n, k, S}\right)$ can be exactly described by an inequality system $\boldsymbol{M r}_{\text {lin,k,s }} \leq \boldsymbol{n}$ generated by strip constraints (6), where the matrix $\boldsymbol{M}$ and the vector $\boldsymbol{n}$ are certain and depend on $k^{i}$ and $\boldsymbol{h}^{i}$. In this way, consistency tests for fault detection consist in verifying whether the origin $O$ of the residual space belongs to $S\left(\boldsymbol{r}_{l i n, k, S}\right)$, i.e. the inequality $0 \leq \boldsymbol{n}$ holds.

Nevertheless, if uncertainty bounds are not known, a set-membership parameter estimation procedure, presented in the following section, is needed.

\section{SET-MEMBERSHIP PARAMETER ESTIMATION}

\subsection{Principle}

The problem considered herein is the following: the residual vector $\boldsymbol{r}_{l i n, k, s}$ (5) is affected by bounded uncertainties $v_{k, s}$ assumed to fluctuate inside a timeinvariant bounded domain $S\left(v_{k, s}\right)$. The objective is the computation of this domain, such that residuals are consistent with data and model structure. At first, this step makes it possible to deduce the bounds of the different parameter uncertainties of model (1) (Ploix et al, 1999). In addition, this procedure allows to reduce the overestimation on $S\left(\boldsymbol{r}_{l i n, k, S}\right)$ (due to the linearization procedure) directly by working on residuals instead of model (1) (Adrot et al, 2000c).

The time-invariant domain $S\left(v_{k, s}\right)$ is assumed to be a parallelotope centered on a value $v_{c}$ :

$$
\begin{gathered}
v_{k, s}=v_{c}+\lambda \boldsymbol{T}_{0} v_{k}, \\
v_{k} \in \mathbb{R}^{s_{v}},\left\|v_{k}\right\|_{\infty} \leq 1, \boldsymbol{T}_{0} \in \mathbb{R}^{s_{v} \times s_{v}}, \lambda \in \mathbb{R}^{+} .
\end{gathered}
$$

The normalized vector $v_{k}$ represents mutually independent bounded variables. The fixed matrix $\boldsymbol{T}_{0}$ and the parameter $\lambda \in \mathbb{R}^{+}$impose respectively the shape and the size of the domain $S\left(v_{k, s}\right)$. With this definition, in the fault free case, (5) is expressed as:

$$
\boldsymbol{R}_{0, k, s} v_{k, s}+\boldsymbol{r}_{0, k, s}=0,
$$

and becomes:

$$
\boldsymbol{R}_{v, k, s} v_{c}+\lambda \boldsymbol{R}_{v, k, s} \boldsymbol{T}_{0} v_{k}+\boldsymbol{r}_{0, k, s}=0 .
$$

Notice that the central parameter vector $v_{c}$ is timeinvariant. It can be obtained using a classical estimator by minimizing an $\alpha$-norm of the equation error raised to power $\beta$ :

$$
v_{c}=\arg \left(\min _{\boldsymbol{\theta}_{c}}\left(\sum_{k=1}^{h-2 s}\left\|\boldsymbol{r}_{0, k, s}+\boldsymbol{R}_{\mathrm{v}, k, s} \boldsymbol{v}_{c}\right\|_{\alpha}^{\beta}\right)\right) .
$$

The solution is to compute the coefficient $\lambda$ (when $v_{c}$ and $\boldsymbol{T}_{0}$ are fixed) such that the residual vector $\boldsymbol{r}_{l i n, k, s}$ (5) explains all the observations (in the fault free case) on the time horizon $k \in\{1, \ldots, h\}$. Thus, the origin $O$ must belong to $S\left(\boldsymbol{R}_{0, k, s} v_{k, s}+\boldsymbol{r}_{0, k, s}\right)$. The individual study of each component of (10) leads to $(s+1) s_{r}$ constraints at each time $k \in\{1, \mathrm{~K}, h-2 s\}$ :

$$
\boldsymbol{r}_{\mathrm{v}, k, s}^{i^{T}} \mathrm{v}_{c}+\lambda \boldsymbol{r}_{\mathrm{v}, k, s}^{i^{T}} \boldsymbol{T}_{0} \mathrm{v}_{k}+r_{0, k, s}^{i}=0,\left\|v_{k}\right\|_{\infty} \leq 1,
$$

where $\boldsymbol{r}_{v, k, s}^{i^{T}}$ and $r_{0, k, s}^{i}$ respectively define the $i^{\text {th }}$ row of $\boldsymbol{R}_{v, k, s}$ and the $i^{\text {th }}$ element of $\boldsymbol{r}_{0, k, s}$. By using interval analysis (Moore, 1979; Ploix et al, 1999), relation (12) leads to following two-sides constraints:

$$
-\lambda\left\|\boldsymbol{r}_{v, k, s}^{i^{T}} \boldsymbol{T}_{0}\right\|_{1} \leq r_{0, k, s}^{i}+\boldsymbol{r}_{v, k, s}^{i^{T}} \mathrm{v}_{c} \leq \lambda\left\|\boldsymbol{r}_{v, k, s}^{i^{T}} \boldsymbol{T}_{0}\right\|_{1}
$$

Therefore, the parameter $\lambda$ satisfies inequalities (14) for all $k \in\{1, \mathrm{~K}, h-2 s\}$ and $i \in\left\{1, \mathrm{~K},(s+1) s_{r}\right\}$ :

$$
\lambda \geq \max \left(0, \frac{\left|r_{0, k, s}^{i}+\boldsymbol{r}_{v, k, s}^{i^{T}} \mathrm{v}_{c}\right|}{\left\|\boldsymbol{r}_{v, k, s}^{i^{T}} \boldsymbol{T}_{0}\right\|_{1}}\right) .
$$

Constraints (13) define the axis-aligned orthotope which is circumscribed to $S\left(\boldsymbol{R}_{v, k, s} v_{k, s}+\boldsymbol{r}_{0, k, s}\right)$. Now, the objective is to take into account dependencies between different equations (12), $i \in\left\{1, \mathrm{~K},(s+1) s_{r}\right\}$, in order to work exactly on the parallelotope $\mathrm{S}\left(\boldsymbol{R}_{\mathrm{v}, k, s} \mathrm{v}_{k, s}+\boldsymbol{r}_{0, k, s}\right)$.

From expression (10), the matrix $\boldsymbol{R}_{k}(\lambda)$ associated with parameter uncertainties $v_{k}$ is defined:

$$
\boldsymbol{R}_{k}(\lambda)=\lambda \boldsymbol{R}_{v, k, s} \boldsymbol{T}_{0}
$$

The method proposed in the following is based on the results detailed in section 3.3, by replacing the matrix $\boldsymbol{R}_{v, k, s}$ by $\boldsymbol{R}_{k}(\lambda)$. Let us note by $\boldsymbol{e}^{l}, l \in\left\{1, \mathrm{~K}, s_{v}\right\}$, the vectors of the canonical basis of $\mathbb{R}^{s_{v}}$. Then, the 
following matrices (see (7)) are built:

$$
\boldsymbol{R}_{k}^{i}(\lambda)=\boldsymbol{R}_{k}(\lambda)\left[\begin{array}{lll}
\boldsymbol{e}^{i_{1}} & \mathrm{~K} & \left.\boldsymbol{e}^{i_{(s+1) s_{r}-1}}\right], \begin{array}{l}
i_{j} \in\left\{1, \mathrm{~K}, s_{v}\right\} \\
j \in\left\{1, \mathrm{~K},(s+1) s_{r}-1\right.
\end{array} .
\end{array} .\right.
$$

If the rank of $\boldsymbol{R}_{k}^{i}(\lambda)$ is equal to $(s+1) s_{r}-1$, an orthogonal row vector $\boldsymbol{h}_{k}^{i^{T}}$ such that $\boldsymbol{h}_{k}^{i^{T}} \boldsymbol{R}_{k}^{i}(\lambda)=0$ is computed. In fact, due to the particular structure of $\boldsymbol{R}_{k}(\lambda)$, the parameter $\lambda$ does not modify the rank of $\boldsymbol{R}_{k}^{i}(\lambda)$ when it is different from 0 (that is to say when some parameter uncertainties exist). Since $\lambda$ is unknown during this step, the projection row vector is found by imposing arbitrary $\lambda=1$ and working on $\boldsymbol{R}_{k}^{i}(1)$ instead of $\boldsymbol{R}_{k}^{i}(\lambda)$. Let $n_{k}$ be the number of vectors $\boldsymbol{h}_{k}^{i}$ obtained by using the previous method.

After multiplying (10) by a row vector $\boldsymbol{h}_{k}^{i^{T}}$, interval analysis leads to the following two-sides inequality:

$$
-\left\|\boldsymbol{h}_{k}^{i^{T}} \boldsymbol{R}_{k}(\lambda)\right\|_{1} \leq \boldsymbol{h}_{k}^{i^{T}}\left(\boldsymbol{r}_{0, k, s}+\boldsymbol{R}_{\mathrm{v}, k, s} v_{c}\right) \leq\left\|\boldsymbol{h}_{k}^{i^{T}} \boldsymbol{R}_{k}(\lambda)\right\|_{1}
$$

At the time $k,(15)$ defines one of the strip constraints describing $\boldsymbol{S}\left(\boldsymbol{R}_{\mathrm{v}, k, s} v_{k, s}+\boldsymbol{r}_{0, k, s}\right)$ and leads to:

$$
\lambda \geq \max \left\{0, \frac{\left|\boldsymbol{h}_{k}^{i^{T}}\left(\boldsymbol{r}_{0, k, s}+\boldsymbol{R}_{\mathrm{v}, k, s} v_{c}\right)\right|}{\left\|\boldsymbol{h}_{k}^{i^{T}} \boldsymbol{R}_{\mathrm{v}, k, s} \boldsymbol{T}_{0}\right\|_{1}}\right\}, \forall i \in\left\{1, \mathrm{~K}, n_{k}\right\} .
$$

At the time $k$, the parameter $\lambda$ has to verify an inequality system composed of the $(s+1) s_{r}-1$ constraints (14) and the $n_{k}$ other ones (16). In fact, the value of the coefficient $\lambda$ imposes the volume of $\mathrm{S}\left(\boldsymbol{R}_{v, k, s} v_{k, s}+\boldsymbol{r}_{0, k, s}\right)$. Therefore, in order to obtain the most precise domain (i.e. the smallest one), $\lambda$ must be minimized. Thus, by assuming that the coefficient $v_{c}$ is fixed, the optimal value of the positive real parameter $\lambda$ corresponds to the minimal value of $\lambda$ satisfying the previous constraints for every index $k$ on the time horizon $h-2 s$ :

$$
\begin{aligned}
\lambda=\underbrace{\sup }_{\forall k \in\{1, \mathrm{~L}, h-2 s\}}\left\{\operatorname { m a x } \left(0, \mathrm{~L} \frac{\left|r_{0, k, s}^{j}+\boldsymbol{r}_{v, k, s}^{j^{T}} \mathrm{v}_{c}\right|}{\left\|\boldsymbol{r}_{v, k, s}^{j^{T}} \boldsymbol{T}_{0}\right\|_{1}} \mathrm{~L}\right.\right. \\
\left.\left.\mathrm{L} \frac{\left|\boldsymbol{h}_{k}^{i^{T}}\left(\boldsymbol{r}_{0, k, s}+\boldsymbol{R}_{\mathrm{v}, k, s} \mathrm{v}_{c}\right)\right|}{\left\|\boldsymbol{h}_{k}^{i^{T}} \boldsymbol{R}_{v, k, s} \boldsymbol{T}_{0}\right\|_{1}} \mathrm{~L}\right)\right\} \\
\forall j \in\left\{1, \mathrm{~L},(s+1) s_{r}\right\}, \forall i \in\left\{1, \mathrm{~L}, n_{k}\right\} .
\end{aligned}
$$

As explained in (Ploix et al., 1999), by taking (11) as initial condition, $v_{c}$ can be optimized by using an additional level of minimization based on a simplex algorithm. Thus, the optimized criterion $J$ is defined by the sum of the volumes of $S\left(\boldsymbol{R}_{v, k, s} v_{k, s}+\boldsymbol{r}_{0, k, s}\right)$ (Lasserre, 1983) on the horizon $h-2 s$ :

$$
J=\sum_{k=1}^{h-2 s} \operatorname{vol}\left(\varsigma\left(\boldsymbol{R}_{v, k, s} \mathrm{v}_{k, s}+\boldsymbol{r}_{0, k, s}\right)\right) .
$$

\subsection{Fault detection}

The principle of consistency tests is explained in sections 2.3. This test checks whether the origin $O$ of the residual space belongs to $S\left(\boldsymbol{r}_{l i n, k}\right)$. Let us notice $\lambda$ ' the value obtained during the parameter estimation when temporal dependence is not taken into account (by applying the previous method on $\boldsymbol{r}_{\text {lin,k }}$ instead of $\boldsymbol{r}_{\text {lin, } k, s}$ and imposing the same center $v_{c}$ ). Then $\lambda^{\prime} \leq \lambda$ since omitted dependencies entertain additional constraints increasing the value of $\lambda$. Therefore, testing whether the origin $O$ of $\mathbb{R}^{s_{r}}$ belongs to $S_{\lambda}\left(\boldsymbol{r}_{\text {lin,k }}\right)$ instead of $S_{\lambda^{\prime}}\left(\boldsymbol{r}_{\text {lin,k }}\right)$ reduces the fault detection quality since $S_{\lambda^{\prime}}\left(\boldsymbol{r}_{\text {lin }, k}\right) \subset S_{\lambda}\left(\boldsymbol{r}_{\text {lin }, k}\right)$. Thus, consistency tests must be modified in order to exploit parameter estimation results: it is needed to test whether the origin of $\mathbb{R}^{(s+1) s_{r}}$ belongs to $S\left(\boldsymbol{r}_{\text {lin,k,s}}\right)$, i.e. $\boldsymbol{S}\left(\boldsymbol{R}_{\mathrm{v}, k, s} \mathrm{v}_{k, s}+\boldsymbol{r}_{0, k, s}\right)$.

At each instant $k$, strip constraints defining $S\left(\boldsymbol{r}_{\text {lin, } k, s}\right)$ are given by (13) and (15) adapted to the fact that a fault may be present (i.e. $\boldsymbol{r}_{l i n, k, s}$ is not necessary equal to 0$)$ with the couple $\left(v_{c}, \lambda\right)$ computed in the section 4.1:

$$
\begin{gathered}
r_{l i n, k, s}^{j} \leq r_{0, k, s}^{j}+\boldsymbol{r}_{v, k, s}^{j^{T}} \mathrm{v}_{c}+\lambda\left\|\boldsymbol{r}_{v, k, s}^{j^{T}} \boldsymbol{T}_{0}\right\|_{1}, \\
-r_{l i n, k, s}^{j} \leq-r_{0, k, s}^{j}-\boldsymbol{r}_{v, k, s}^{j^{T}} v_{c}+\lambda\left\|\boldsymbol{r}_{v, k, s}^{j^{T}} \boldsymbol{T}_{0}\right\|_{1} \\
\boldsymbol{h}_{k}^{i^{T}} \boldsymbol{r}_{l i n, k, s} \leq \boldsymbol{h}_{k}^{i^{T}}\left(\boldsymbol{r}_{0, k, s}+\boldsymbol{R}_{\mathrm{v}, k, s} \mathrm{v}_{c}\right)+\lambda\left\|\boldsymbol{h}_{k}^{i^{T}} \boldsymbol{R}_{\mathrm{v}, k, s} \boldsymbol{T}_{0}\right\|_{1} \|^{\prime} \\
-\boldsymbol{h}_{k}^{i^{T}} \boldsymbol{r}_{l i n, k, s} \leq-\boldsymbol{h}_{k}^{i^{T}}\left(\boldsymbol{r}_{0, k, s}+\boldsymbol{R}_{\mathrm{v}, k, s} \mathrm{v}_{c}\right)+\lambda\left\|\boldsymbol{h}_{k}^{i^{T}} \boldsymbol{R}_{\mathrm{v}, k, s} \boldsymbol{T}_{0}\right\| \\
\forall j \in\left\{1, \mathrm{~L},(s+1) s_{r}\right\} \forall i \in\left\{1, \mathrm{~L}, n_{k}\right\} .
\end{gathered}
$$

At the end, $\boldsymbol{S}\left(\boldsymbol{r}_{l i n, k, S}\right)$ is exactly described by the system $\boldsymbol{M r}_{l i n, k, s} \leq \boldsymbol{n}$ generated by previous inequalities. In this way, consistency tests for fault detection consist in verifying whether $0 \leq \boldsymbol{n}$ holds.

\section{EXAMPLE}

In order to illustrate previous developments, let us consider the following state representation:

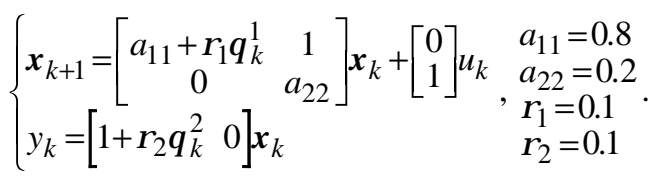


Normalized bounded variables $\theta_{k}^{i}, i \in\{1,2\}$, describe multiplicative uncertainties. The chosen time horizon (i.e. the smallest integer $s$ for which $\boldsymbol{O}_{s}$ is not full row rank) is $s=2$, what leads to an alone residual $r_{k}$ (3). Then the linearization procedure is applied and the residual $r_{l i n, k}(4)$ is built. Even if the chosen model is simple, 15 bounded variables intervene in $r_{l i n, k}$ : $\mu_{k} \in \mathbb{R}^{15}$. Due to temporal dependencies, $r_{\text {lin, } k}$ is stacked on the time horizon $[k, k+2]$ and the residual vector $\boldsymbol{r}_{\text {lin }, k, 2}$ (5) is obtained: $\boldsymbol{r}_{\text {lin }, k, 2} \in \mathbb{R}^{3}, v_{k, 2} \in \mathbb{R}^{35}$. In order to show residual structure, only the three first terms of $\boldsymbol{r}_{l i n, k, 2}$ are detailed:

$$
\boldsymbol{r}_{\text {lin }, k, 2}=\left[\begin{array}{ccc}
a_{22} \rho_{1} & -\rho_{1} y_{k+1} & 0 \\
0 & a_{22} \rho_{1} y_{k+1} & -\rho_{1} \rho_{2} y_{k+2} L \\
0 & 0 & a_{22} \rho_{1} \rho_{2} y_{k+2}
\end{array}\right] v_{k, s}+r_{0, k, s} .
$$

For a chosen center $v_{c}$ which coincides with the origin of the parameter space, the set-membership parameter estimation gives $\lambda=0.76$. This value shows that the linearization procedure has entertained an important overestimation of the normal operation domain since $S_{\lambda=0.76}\left(\boldsymbol{r}_{\text {lin }, k, 2}\right) \subset S_{\lambda=1}\left(\boldsymbol{r}_{\text {lin }, k, 2}\right)$. Checking whether the origin $O$ of the residual space belongs to $S_{\lambda=0.76}\left(\boldsymbol{r}_{l i n, k, 2}\right)$ is more precise than the same operation with $S_{\lambda=1}\left(\boldsymbol{r}_{l i n, k, 2}\right)$.

In figure 3 , the parallelotope $S_{\lambda=0.76}\left(\boldsymbol{r}_{\text {lin, } k, 2}\right)$ is represented at a particular instant $k$ (sample $k=43)$. To realize consistency tests without taking into account temporal dependencies would consist in using the axis-aligned orthotope (in gray in figure 3) circumscribed to the value set $S_{\lambda=0.76}\left(\boldsymbol{r}_{l i n, k, 2}\right)$. This orthotope may entertain some no-detections since $O$ may be inside this one whereas it may not belong to $S_{\lambda=0.76}\left(\boldsymbol{r}_{\text {lin, } k, 2}\right)$.

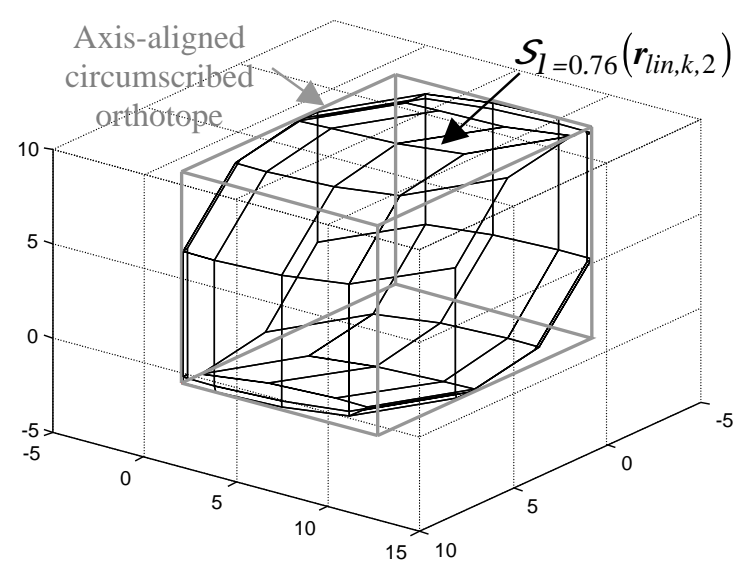

Fig 3. Value set of $\boldsymbol{r}_{\text {lin, } k, 2}$ for $k=43$

The system is simulated by adding two multiplicative faults and 200 observations are generated. For observations, which index belongs to $[20,70]$ and $[140,190]$ (gray areas in figure 4 ), $\theta_{k}^{1}$ is equal to a bias of magnitude 2 . The results of the proposed fault detection procedure are presented in figure 4, where the value 1 corresponds to an inconsistency. The fault detection depends on operation points and unknown uncertain parameter values, thus sometimes, $S_{\lambda=0.76}\left(\boldsymbol{r}_{\text {lin,k,2 }}\right)$ contains $O$ even if a fault is present. But globally, faults are well detected and the setmembership parameter estimation is conclusive since no false alarm is present.

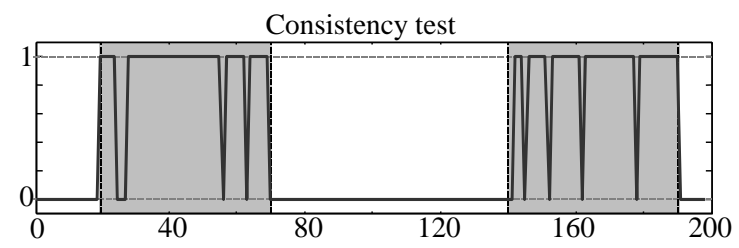

Fig 4. Consistency test

\section{CONCLUSION}

Against to our previous works on fault diagnosis using interval analysis, the method proposed in this paper takes into account temporal dependence between residuals. This additional information increases time consuming since a bigger number of bounded variables intervene in residuals, but the precision of the fault detection procedure is theoretically improved. Notice that for complicated models, this method becomes problematic because of the number of bounded variables to treat.

\section{REFERENCES}

Adrot, O., D. Maquin and J. Ragot, (1999). Fault detection with model parameter structured uncertainties, $E C C^{\prime} 99$.

Adrot, O., D. Maquin and J. Ragot, (2000a). Bounding approach to the fault detection of uncertain dynamic systems, Safeprocess 2000.

Adrot, O., D. Maquin and J. Ragot (2000b). Diagnosis of an uncertain static system, 39th CDC'2000.

Adrot, O., (2000c). Diagnostic à base de modèles incertains utilisant l'analyse par intervalles: l'approche bornante, PhD of "Institut National Polytechnique de Lorraine”, France.

Armengol, J., L. Travé-Massuyés, J. Vehi and J.L. De la Rosa (1999). A survey on interval model simulators and their properties related to fault detection, IFAC Beijing'99.

Lasserre, J.B. (1983). An analytical expression and an algorithm for the volume of a convex polyhedron in $\mathbb{R}^{\mathrm{n}}$, Journal of Optim. Theory and Applications, vol 39, pp 363-377.

Moore, R.E., (1979). Methods and applications of interval analysis, SIAM, Philadelphia.

Ploix, S., O. Adrot and J. Ragot (1999). Parameter uncertainty computation in static linear models, CDC'99.

Ploix, S., O. Adrot and J. Ragot, (2000). Bounding approach to the diagnosis of uncertain static systems, Safeprocess 2000. 